\title{
Time to Bring Single Neuron Modeling into 3D
}

\author{
Weiliang Chen ${ }^{1} \cdot$ Erik De Schutter $^{1}$ (D)
}

Published online: 5 January 2017

(C) Springer Science+Business Media New York 2017

Almost all single neuron models currently being used and developed combine discretized cable models with HodgkinHuxley type equations. ${ }^{1}$ This approach, employed by the popular software package NEURON ${ }^{2}$ and its competitors such as GENESIS, ${ }^{3}$ MOOSE, ${ }^{4}$ etc., is based on mathematical methods developed in the 50s and 60s, before supercomputers were available for scientific research. Wilfrid Rall introduced linear cable theory to analyze the electrical behavior of dendrites $^{5}$ and was the first to demonstrate their importance for synaptic integration. ${ }^{6}$ Later he proposed a computational approach, called compartmental modeling, based on a spatial discretization of the linear cable equation. ${ }^{7}$ Although compartmental modeling allows simulation of the complex 3D

\footnotetext{
${ }^{1}$ Koch, C., \& Segev, I. (Ed.) (1998). Methods in neuronal modeling: from ions to networks. MIT Press.

${ }^{2}$ Carnevale, N. T., \& Hines, M. L. (2006). The Neuron Book. Cambridge University Press.

${ }^{3}$ Bower, J. M., \& Beeman, D. (1998). The book of GENESIS: exploring realistic neural models with the GEneral NEural SImulation System. Springer-Verlag, New York.

${ }^{4}$ Bhalla, U. S. (2008). PyMOOSE: interoperable scripting in Python for MOOSE. Frontiers in Neuroinformatics, 2, 6

${ }^{5}$ Rall, W. (1959). Branching dendritic trees and motoneuron membrane resistivity. Experimental Neurology, 1, 491-527.

${ }^{6}$ Rall, W. (1962). Theory of physiological properties of dendrites. Annals of the New York Academy of Sciences, 96, 1071-1092.

${ }^{7}$ Rall, W. (1964). Theoretical significance of dendritic tree for input-output relation. In R. F. Reiss (Ed.), Neural Theory and Modeling (pp. 73-97). Stanford University Press.
}

Erik De Schutter erik@oist.jp

1 Computational Neuroscience Unit, Okinawa Institute of Science and Technology Graduate University, Onna, Japan morphology of dendrites and axons in reasonable detail, the underlying equations ignore many $3 \mathrm{D}$ aspects by computing the changes in membrane potential in a branched 1D cable. Each cylindrical compartment is isopotential with a constant radius and uniform membrane currents. In some cases the use of tapering cylinders ${ }^{2}$ can provide a better approximation of the real neuronal shape.

Compartmental models can easily be extended to include nonlinear conductances by adding Hodgkin-Huxley type equations ${ }^{8}$ to represent different types of voltage/ligandgated channels. This method is extensible and in principle all types of channels identified can be included in a model, provided the necessary kinetic data are available for the Hodgkin-Huxley type equations. ${ }^{9}$ For most neuron types the cytoplasmic calcium concentrations will also need to be computed to simulate calcium-activated potassium channels. ${ }^{10} \mathrm{~A}$ simple well-mixed solution, usually called the calcium pool, is often used but this is not effective to simulate the multiple time constants by which calcium concentrations can evolve. ${ }^{11}$ More realistic models of calcium dynamics require calcium diffusion and here one runs into limitations of the compartmental approach. Because a uniform membrane current is assumed, the only gradient for calcium within each compartment is perpendicular to the membrane and therefore only $1 \mathrm{D}$

\footnotetext{
${ }^{8}$ Hodgkin, A. L., \& Huxley, A. F. (1952). A quantitative description of membrane current and its application to conduction and excitation in nerve. The Journal of Physiology, 117(4), 500-544.

${ }^{9}$ De Schutter, E., \& Van Geit, W. (2009). Modeling complex neurons. In E. De Schutter (Ed.), Computational Modeling Methods for Neuroscientists (pp. 259-283). MIT Press.

${ }^{10}$ De Schutter, E. (2009). Modeling intracellular calcium dynamics. In E. De Schutter (Ed.), Computational Modeling Methods for Neuroscientists (pp. 114). MIT Press.

${ }^{11}$ Anwar, H., Hong, S., \& Schutter, E. (2010). Controlling $\mathrm{Ca}^{2+}$-Activated $\mathrm{K}^{+}$ channels with models of $\mathrm{Ca}^{2+}$ buffering in purkinje cells. Cerebellum (London, England), 11(3), 681-693.
} 


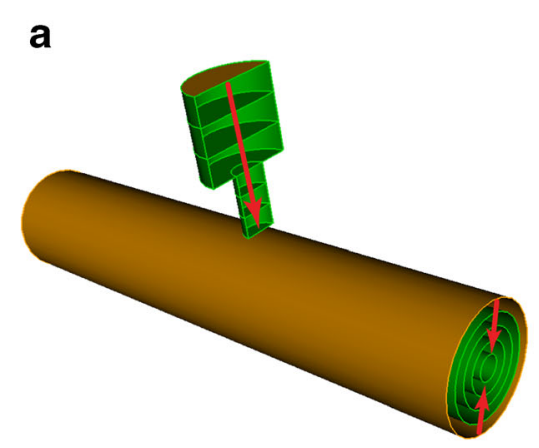

Fig. 1 a. 1D calcium diffusion in compartmental models of a spine (top) and of an unbranched dendrite (bottom). In each green section the calcium concentration is well-mixed. Red arrows show direction of calcium diffusion, brown membrane represents the site of homogenous calcium influx. Notice that the spine does not 'fit' onto the dendrite because of their different shapes. Also, the calcium concentration gradients in the dendritic compartment ignore the position of the spine if the two systems

calcium diffusion can be simulated, usually radially if the goal is to model potassium channel activation ${ }^{12}$ (Fig. 1a, bottom). In addition, calcium gradients occur over much shorter distances than voltage gradients due to strong calcium buffering in most neurons and therefore a reasonably accurate model of longitudinal calcium gradients will require very short electrical compartments. ${ }^{13}$ More challenging is to model calcium influx through synaptic channels in a dendritic spine. A common approach is to model the spine head as a stack of short cylinders, attached to a similar stack of narrower cylinders representing the spine neck, and simulate the 1D diffusion along the longitudinal axis of this system (Fig. 1a, top). This works fine until one tries to attach this spine to a dendrite using radial diffusion: the morphologies do not connect properly. It is difficult to compute the volumes at the connection and it requires very short dendritic compartments to represent the localized effects of calcium changes in spines (Fig. 1a).

In general, compartmental modeling does a poor job of simulating structures that do not look like cylinders, such as somas, axonal boutons or spines. It is also difficult to compute the effects of small changes in shape, like the enlargement of spines during induction of long-term potentiation, ${ }^{14}$ if the morphology has been approximated. In the past the limitations of the cylindrical model were not a big concern because reconstructions of neural morphology were also imprecise. The standard manual approach to neural reconstruction, as

\footnotetext{
${ }^{12}$ De Schutter, E., \& Smolen, P. (1998). Calcium dynamics in large neuronal models. In . C. Koch and I. Segev (Eds.) Methods in Neuronal Modeling: From Ions to Networks (pp. 211-250). MIT Press.

${ }^{13}$ Anwar, H., Roome, C. J., Nedelescu, H., Chen, W., Kuhn, B., \& De Schutter, E. (2014). Dendritic diameters affect the spatial variability of intracellular calcium dynamics in computer models. Frontiers in Cellular Neuroscience, 8,168

${ }^{14}$ Matsuzaki, M., Honkura, N., Ellis-Davies, G. C. R., \& Kasai, H. (2004). Structural basis of long-term potentiation in single dendritic spines. Nature, 429(6993), 761-766.
}

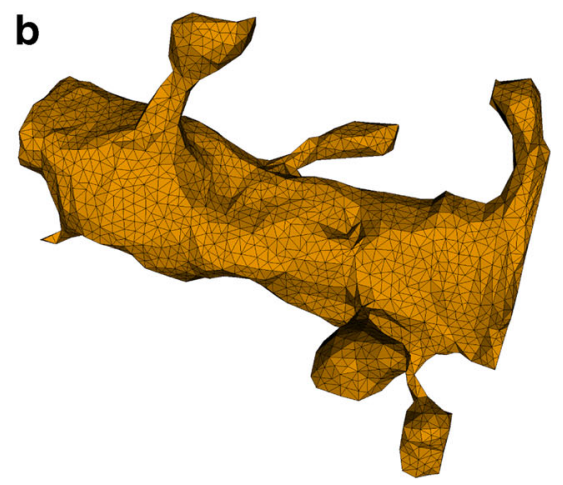

were to be connected. $\mathbf{b}$. Tetrahedral mesh of part of a spiny dendrite CA1 pyramidal neuron (Harris, K. M., \& Stevens, J. K. (1989). Dendritic spines of CA 1 pyramidal cells in the rat hippocampus: serial electron microscopy with reference to their biophysical characteristics. The Journal of Neuroscience, 9(8), 2982-2997). In this representation calcium diffusion and electrical events can be simulated in 3D at the resolution of the mesh elements

exemplified by the Neurolucida software, describes the neuron as a branched cable with slowly changing diameters and is limited by the poor resolution of light microscopy below $0.5 \mu \mathrm{m} .{ }^{15}$ With the increased use of electron microscopy ${ }^{16}$ and innovations like super resolution microscopy ${ }^{17}$ much better neural reconstructions are becoming available that often deviate from the cylinder assumption. For example, many dendrites have an elliptical cross section instead of a circular one. ${ }^{18}$ High resolution reconstruction data is typically described by a surface mesh (Fig. 1b), which provides much more detail than a traditional reconstruction, as found on neuromorpho.org. ${ }^{19}$

Linear cable theory and compartmental modeling assume that the resistance of the external medium is uniform and usually ignore it $\left(r_{e}=0\right)^{5}$. This assumption does not matter much for membrane potential calculations but limits the validity of models of the extracellular local field potential (LFP). Recent studies show a strong interest in modeling approaches to understand the source of the LFP and to interpret the increasing number of experiments where LFP is recorded at multiple sites. ${ }^{20}$ Such simulations are typically based on

\footnotetext{
15 Jaeger, D. (2000). Accurate reconstruction of neuronal morphology. In E. De Schutter (Ed.), Computational neuroscience: Realistic modeling for experimentalists (pp. 159-178). CRC Press.

${ }^{16}$ Jacobs, G. A., Claiborne, B., \& Harris, K. M. (2009). Reconstruction of neuronal morphology. In E. De Schutter (Ed.), Computational Modeling Methods for Neuroscientists (pp. 187-210). MIT Press.

${ }^{17}$ Yamanaka, M., Smith, N. I., \& Fujita, K. (2014). Introduction to superresolution microscopy. Microscopy, 63(3), 177-192.

${ }^{18}$ Kubota, Y., Karube, F., Nomura, M., Gulledge, A. T., Mochizuki, A., Schertel, A., \& Kawaguchi, Y. (2011). Conserved properties of dendritic trees in four cortical interneuron subtypes. Scientific Reports, 1, 89.

${ }^{19}$ Nanda, S., Allaham, M. M., Bergamino, M., Polavaram, S., Armañanzas, R., Ascoli, G. A., \& Parekh, R. (2015). Doubling up on the fly: NeuroMorpho.Org Meets Big Data. Neuroinformatics, 13(1), 127-129.

${ }^{20}$ Einevoll, G. T., Kayser, C., Logothetis, N. K., \& Panzeri, S. (2013). Modelling and analysis of local fieldpotentials for studying the function ofcortical circuits. Nature Reviews Neuroscience, 14(11), 770-785.
} 
networks of compartmental models of neurons of varying morphological detail. ${ }^{21}$ Because the physical mechanisms causing the LFP are a topic of active discussion ${ }^{22}$ it is at present difficult to predict the major sources of error in this modeling approach. Nevertheless, it is clear that the use of uniform membrane currents on cylinders and the assumption of a homogeneous extracellular medium are gross simplifications, especially in the presence of tightly packed neurons.

Considering the many limitations of compartmental modeling mentioned, the lack of development of alternative methods to simulate morphologically detailed neurons is surprising. A promising effort was the NeuroDune project, ${ }^{23}$ which used finite volume approaches to accurately model the $3 \mathrm{D}$ placement of synapses with the neuron model still based on cable theory. Unfortunately this project seems no longer active and its website is defunct. Our group has been developing a software package STEPS for the stochastic simulation of reactiondiffusion systems in neurons. ${ }^{24}$ STEPS uses tetrahedral volume meshes to represent the full 3D morphology with an accuracy of $0.1 \mu \mathrm{m}$ and below (Fig. 1b). Like several other software packages used to model molecular events in synap$\mathrm{ses},{ }^{25}$ STEPS was originally designed to simulate only small parts of neurons, such as spines ${ }^{26}$ or partial dendrites. ${ }^{27}$ Thanks to a recent MPI-based parallel version with supralinear scaling on computing clusters, ${ }^{28}$ simulation of complete neurons in 3D is now practical with STEPS. This allows for detailed comparison with compartmental models ${ }^{13}$ but also, and more interestingly, it makes molecular level simulation at the full neuronal scale possible. An example is simulation of the effect of different clustering schemes for calcium and calcium-activated potassium channels at the sub- $\mu \mathrm{m}$ scale act on the generation of dendritic calcium spikes ${ }^{27}$. Nevertheless this is not a complete solution: not every problem requires computationally intensive stochastic simulation and at present STEPS does not support network simulation or computation of the LFP.

The number of scientific questions at the molecular, structural and network levels that cannot be properly simulated with a $1 \mathrm{D}$ approach is expected to increase. With easier access to large computing power provided by university clusters and cloud computing, there is no longer a need for the numerical efficiency of cable theory to model a single neuron. Meshbased simulators that fully represent the shape of neurons at high resolution and compute electrical events in $3 \mathrm{D}$ can run on such computing platforms at good speeds. This approach may not be practical yet for large neural network simulations, ${ }^{29}$ but it would serve the computational neuroscience community well to recognize the limitations of cable theory and foster the development of several new software platforms to simulate neuronal physiology in 3D.

\footnotetext{
${ }^{21}$ Głąbska, H., Chintaluri, C., \& Wójcik, D. K. (2017). Collection of simulated data from a thalamocortical network model. Neuroinformatics, 15.

22 Destexhe, A., \& Bedard, C. (2012). Do neurons generate monopolar current sources? Journal of Neurophysiology, 108(4), 953-955; Gratiy, S. L., Pettersen, K. H., Einevoll, G. T., \& Dale, A. M. (2013). Pitfalls in the interpretation of multielectrode data: on the infeasibility of the neuronal currentsource monopoles. Journal of Neurophysiology, 109(6), 1681-1682.

${ }^{23}$ Lang, S., Dercksen, V. J., Sakmann, B., \& Oberlaender, M. (2011). Simulation of signal flow in 3D reconstructions of an anatomically realistic neural network in rat vibrissal cortex. Neural Networks, 24(9), 998-1011.

${ }^{24}$ Hepburn, I., Chen, W., Wils, S., \& De Schutter, E. (2012). STEPS: efficient simulation of stochastic reaction-diffusion models in realistic morphologies. BMC Systems Biology, 6, 36.

${ }^{25}$ Bartol, T. M., Jr., Keller, D. X., Kinney, J. P., Bajaj, C. L., Harris, K. M., Sejnowski, T. J., \& Kennedy, M. B. (2015). Computational reconstitution of spine calcium transients from individual proteins. Frontiers in Synaptic Neuroscience, 7. 00017; Kotaleski, J. H., \& Blackwell, K. T. (2010). Modelling the molecular mechanisms of synaptic plasticity using systems biology approaches. Nature Reviews Neuroscience, 11(4), 239-251.

${ }^{26}$ Simon, C. M., Hepburn, I., Chen, W., \& De Schutter, E. (2014). The role of dendritic spine morphology in the compartmentalization and delivery of surface receptors. Journal of Computational Neuroscience 36, 483-497.

${ }^{27}$ Anwar, H., Hepburn, I., Nedelescu, H., Chen, W., \& De Schutter, E. (2013). Stochastic calcium mechanisms cause dendritic calcium spike variability. The Journal of Neuroscience, 33(40), 15848-15867.

${ }^{28}$ Hepburn, I., Chen, W., \& De Schutter, E. (2016). Accurate reactiondiffusion operator splitting on tetrahedral meshes for parallel stochastic molecular simulations. The Journal of Chemical Physics, 145(5), 054118.
}

\footnotetext{
${ }^{29}$ Markram, H., Muller, E., Ramaswamy, S., Reimann, M. W., Abdellah, M., Sanchez, C. A., et al. (2015). Reconstruction and simulation of neocortical microcircuitry. Cell, 163(2), 456-492.
} 